\title{
25 Research Square \\ Selective Ultrasound Guided Nerve Blocks to Diagnose Anteromedial Neuropathic Knee Pain
}

\section{Sabine Popp ( $\nabla$ sabine.popp@gmx.at)}

Hospital of the Brothers of Saint John of God, Department of Diagnostic and Interventional Radiology

Christopher Pivec

PUC Private Ultrasound Center Wien

\section{Gregor Drlicek}

Medical University of Vienna, Department of Anaesthesia, General Intensive Care Medicine and Pain

Medicine

Janos Gellen

PUC Private Ultrasound Center Graz

Georg Riegler

PUC Private Ultrasound Center Graz

\section{Research Article}

Keywords: Chronic neuropathic pain, Sonomorphologic changes, HRUS, anteromedial knee, electronical medical records

Posted Date: January 20th, 2021

DOI: https://doi.org/10.21203/rs.3.rs-136683/v1

License: (c) (i) This work is licensed under a Creative Commons Attribution 4.0 International License.

Read Full License 


\section{Abstract}

Purpose: Chronic neuropathic pain, due to injury to the cutaneous nerve branches after knee surgery, is a common postoperative problem. Therefore, the aim of this retrospective study was to evaluate the ability of High-resolution Ultrasound (HRUS) to correctly diagnose the cause and origin of neuropathic pain using selective ultrasound-guided diagnostic blocks.

Methods: Data were obtained retrospectively (time frame 2011-2019) from a consecutive cohort of patients through a review of electronical medical records. Patients with postoperative neuropathic knee pain and consecutive HRUS-guided selective nerve block were included. The main outcome measure was pain intensity changes (baseline and post-blockade). A block was rated positive if there was $\geq 50 \%$ pain relief. Further, ultrasound findings suggesting nerve damage or involvement were assessed.

Results: In 80/116 (69\%) performed examinations, the diagnostic block was positive. Sonomorphologic changes were detected in $64 / 116$ cases $(55.2 \%)$, including neuromas $21 / 116(18.1 \%)$, nerve thickening $12 / 116(10.3 \%)$, scar tissue $26 / 116$ (22.4\%), and involvement by other structures, e.g., ganglia 5/116 (4.3\%).

Conclusion: HRUS and selective ultrasound-guided nerve blocks are valuable tools for the diagnosis of neuropathic pain due to cutaneous nerve injury at the anteromedial knee after surgery.

\section{Introduction}

Postoperative, chronic, neuropathic knee pain due to injuries to the small cutaneous nerve branches is a well-known clinical problem, accounting for discomfort in up to $13 \%$ of patients six months after total knee arthroplasty 123 .

Mostly, clinical symptoms after cutaneous nerve damage are mild, such as irritating paresthesia or hypesthesia ${ }^{4} 5$. Nevertheless, in some patients, severe chronic pain that leads to obviously reduced quality of life have also been described ${ }^{6}$.

Most surgical approaches at the knee are performed at the anteromedial knee ${ }^{7}$. In case of iatrogenic injuries, cutaneous nerve branches that innervate this area, such as the saphenous nerve, the intrapatellar branches of the saphenous nerve, and the anterior femoral cutaneous branches of the femoral nerve, may be affected and could be a potential source of neuropathic pain 89 .

To correctly identify neuropathic pain, the quality and occurrence of the pain, as well as the distribution patterns of the various nerves, must be considered ${ }^{10}$. Since the variability of the nerve branches and the cutaneous peripheral nerve fields at the anteromedial knee vary extensively, a sufficient diagnosis based only on clinical symptoms and examination may not be accurate ${ }^{11}$. 
Since high-resolution ultrasound (HRUS), using high-frequency probes, offers excellent tissue differentiation for the examination of small cutaneous nerve branches, and thus, enables the correct selective nerve blocks, it is most important to correctly diagnose the origin of neuropathic pain ${ }^{12}$.

Therefore, the aim of this retrospective study was to evaluate the ability of high-resolution ultrasound (HRUS) to correctly diagnose the cause and origin of neuropathic pain using selective ultrasound-guided diagnostic blocks, and to assess the morphological tissue changes that lead to neuropathic pain.

\section{Methods}

This study was approved by the Ethics Committee of the Medical University of Vienna. The study was performed in accordance with the World Medical Association Declaration of Helsinki. The Ethics committee of the Medical University of Vienna did not require informed consent for approval of this study due to its retrospective nature.

Data from patients referred to the Department of Biomedical Imaging and Image-guided Therapy at the Medical University of Vienna due to clinically suspected neuropathic pain at the anteromedial knee between the years 2011 and 2019 were collected.

The data system (AKIM) was searched for ultrasound examinations, including the following nerve branches: saphenous nerve (SN); infrapatellar nerve branches of the saphenous nerve (IPBSN); and anterior femoral cutaneous nerve branches (FCN). From this data set, 334 performed ultrasound examinations of the above-mentioned nerves in 184 patients could be identified. The following inclusion and exclusion criteria were applied:

Inclusion criteria

- history of knee or lower limb surgery

- diagnosis by ultrasound-guided diagnostic block

\section{Exclusion criteria}

- duplicate blocks of one nerve per patient

Information about patient characteristics and the performed ultrasound examinations was retrospectively collected. Information about patient characteristics included: age; sex; examined nerve; sonomorphological pathologies; previous operation; local anesthetic drug used for diagnostic block; nerve block response; and further treatment.

A positive block was defined as resulting in a $50 \%$ reduction in pain compared to baseline (prior to the ultrasound-guided block).

\section{Statistical analysis}


Descriptive statistics were performed using IBM SPSS Statistics for Windows Version 26.0.0.0 (IBM, Armonk, NY, USA). Metric data, such as age, are presented as mean (+/- standard deviation; SD) and range (minimum to maximum).

\section{Results}

\section{Patient characteristics:}

The study showed a clear female predominance - 74 female patients (77.1\% female patients) and 22 male patients (22.9\%). There was a female to male ratio of about 3.5:1.

At the time of diagnosis, patients had a mean age of 56.6 years, age range: $15-89$ years.

Of the 116 diagnostic blocks, 80 blocks (69\%) were positive (pain reduction of 50\% or more); 17 were negative (pain reduction less than 50\%), and, in 19 cases, information about the result of the block was not reported. Examples of ultrasound-guided nerve blocks are visualized in Fig. 2.

The diagnostic blocks were performed using lidocaine in 35 cases (30.2\%) and bupivacaine in two cases (1.7\%). In the remaining cases, the local anesthetic was not specified.

To achieve a selective block of each nerve, $0.3 \mathrm{ml}$ of local anesthetic was used in three cases (2.6\%), $0.5 \mathrm{ml}$ in 14 cases (12.1\%), $0.7 \mathrm{ml}$ in three cases (2.6\%), $1 \mathrm{ml}$ in 33 cases (28.4\%), $1.5 \mathrm{ml}$ in three cases (2.6\%), and $2 \mathrm{ml}$ in nine cases (8.8\%). In 51 cases, the amount was not specified.

Sonomorphological pathologic changes of the investigated nerves were described in 64 of the patients. In 21 examinations no pathology was detected and in 31 examinations no pathologies were described. The different sonomorphological changes are listed in Table 1.

Table 1

- Listing of the different sonomorphological changes detected in patients

\begin{tabular}{|lc|}
\hline No pathologies visible & $\mathbf{2 1}$ \\
\hline Neuroma & 21 \\
\hline Nerve thickening & 12 \\
\hline Involvement of nerve by scar tissue & 26 \\
\hline Involvement by other structures (ganglion, etc.) & 5 \\
\hline No pathologies described & 31 \\
\hline
\end{tabular}

The operations in the patients' history prior to ultrasound-guided nerve blocks, 
as well as different ultrasound-guided therapies applied after achieving a positive diagnostic block, are described in Tables 2 and 3. Examples of different morphological nerve changes are visualized in Fig. 3.

Table 2

- Listing of the different surgeries in the patients' history prior to ultrasound-guided nerve blocks

\begin{tabular}{|ll|}
\hline Knee arthroscopy & $\mathbf{2 2}$ \\
\hline Knee arthroplasty & 29 \\
\hline Total hip arthroplasty & 7 \\
\hline Lower limb amputation & 6 \\
\hline Neurolysis & 4 \\
\hline Other & 28 \\
\hline
\end{tabular}

Table 3

- Listing of further applied ultrasound-guided therapies after positive ultrasound-guided block in the patients' history

\begin{tabular}{|ll|}
\hline Radiofrequency ablation & 13 \\
\hline Alcohol sclerosing injections & 9 \\
\hline Hydrodissection & 6 \\
\hline
\end{tabular}

\section{Discussion}

Postoperative chronic pain after knee surgery is a relevant clinical problem ${ }^{13}$. Common underlying causes for postoperative knee pain are infection, loosening of the prosthesis, or instability. ${ }^{814}$. However, intraoperative nerve damage also has to be taken into consideration. In a prospective cohort study by Rice et al. that investigated chronic postoperative pain after total knee arthroplasty, more than half of the affected patients had pain that was most likely neuropathic ${ }^{15}$.

Chronic postoperative pain of the anteromedial knee region is often caused by iatrogenic nerve injury during incisions for different procedures ${ }^{3}$. The saphenous nerve and its infrapatellar branches are often affected during knee operations, such as arthroplasty, arthroscopy, meniscal surgery, and ligamentoplasty, as well as during harvesting of the saphenous vein for coronary bypass graft procedures 1617 . Other nerves commonly at risk during operative procedures of the knee are the intermediate and medial femoral cutaneous nerves, which are two anterior cutaneous branches of the femoral nerve that provide sensory innervation of the medial thigh ${ }^{1810}$. Since these branches show a high variability in their course, especially in the anteromedial knee region, they are at a high risk for injury during incision ${ }^{19}$. 
In our patient collective, the most common procedures in the history of the patients who suffered from chronic postoperative pain were either knee prostheses or knee arthroscopies. A reliable tool for the diagnosis of this pain is necessary since these operations are performed more and more frequently, with a high risk of intraoperative nerve damage, and up to one-fifth of patients suffer from chronic postoperative pain, with a high percentage of neuropathic pain.

As presented in this study, high-resolution ultrasound provides a valuable tool with which to diagnose postoperative pathologic changes of the above-mentioned nerve branches, such as neuromas or nerve thickening. Another relevant finding that can be readily assessed via ultrasound is scar tissue surrounding an affected nerve along its course.

However, the sonomorphological findings are not sufficient to reach a diagnosis in about half of all patients, since pathologic findings were only detected in about $54 \%$ of all cases. Therefore, it is necessary to perform highly selective ultrasound-guided peripheral nerve blocks to help confirm the diagnosis of which nerve is affected in the absence but also in the presence of sonomorphologic changes. To achieve a precise identification of the responsible nerve branch, only small amounts of local anesthesia should be administered. In our study, no more than $2 \mathrm{ml}$ of local anesthesia was injected under ultrasound guidance in any patient. A reduction of at least $50 \%$ of the patient's symptoms had to be achieved for a nerve block to be positive and to establish a definitive diagnosis.

The correct identification of the affected nerve also provides options for further ultrasound-guided treatments, i.e., ultrasound-guided radiofrequency ablation or alcohol sclerosing injections 202122 . Another treatment option is hydrodissection if a nerve is affected by scar tissue along its course. Furthermore, if a surgical treatment option is planned, the altered nerve can be marked preoperatively using high-resolution ultrasound 232425 .

Interestingly, our patient collective showed a clear female to male predominance, with a ratio of female to male of $3.5: 1$. The reason for this is not quite clear; one paper that investigated chronic postoperative pain reported that female gender was a risk factor ${ }^{26}$. Another possible explanation may be that female patients visit the doctor's office more often for pain; however, further exploration into this subject is warranted.

Our study has strengths and limitations. One strength is the large patient collective. The main limitation of this study is that data were collected retrospectively, and therefore, some data were missing, e.g., pain reduction after diagnostic blocks was not reported in all patients.

In conclusion, this study confirms the value of high-resolution ultrasound as a diagnostic tool with which to identify the underlying causes of chronic postoperative pain with higher confidence. In addition, in case of postoperative nerve injury, HRUS allows the physician to reach a definite diagnosis using highly selective peripheral ultrasound-guided nerve blocks in the absence as well as in the presence of sonomorphological changes. 
Furthermore, high-resolution ultrasound aids in patient management via ultrasound-guided treatment options, as well as interdisciplinary therapeutic patient management with other specialities.

\section{Declarations}

\section{Acknowledgments}

The authors thank Mary McAllister for her comments on the manuscript and Aron Cserveny for his help with graphical illustrations.

\section{Competing interests}

The authors declare no competing interests.

\section{References}

1. Fuzier, R., Rousset, J., Bataille, B., Salces-y-Nedeo, A. \& Magues, J.-P. One half of patients reports persistent pain three months after orthopaedic surgery. Anaesthesia, Crit. care pain Med. 34, 159164 (2015).

2. Duale, C., Ouchchane, L., Schoeffler, P. \& Dubray, C. Neuropathic aspects of persistent postsurgical pain: a French multicenter survey with a 6-month prospective follow-up. J. Pain 15, 24.e1-24.e20 (2014).

3. Mistry, D. \& O'Meeghan, C. Fate of the infrapatellar branch of the saphenous nerve post total knee arthroplasty. ANZ J. Surg. 75, 822-824 (2005).

4. Laffosse, J.-M., Potapov, A., Malo, M., Lavigne, M. \& Vendittoli, P.-A. Hypesthesia after anterolateral versus midline skin incision in TKA: a randomized study. Clin. Orthop. Relat. Res. 469, 3154-3163 (2011).

5. Sundaram, R. O., Ramakrishnan, M., Harvey, R. A. \& Parkinson, R. W. Comparison of scars and resulting hypoaesthesia between the medial parapatellar and midline skin incisions in total knee arthroplasty. Knee 14, 375-378 (2007).

6. Hasegawa, M., Tone, S., Naito, Y., Wakabayashi, H. \& Sudo, A. Prevalence of Persistent Pain after Total Knee Arthroplasty and the Impact of Neuropathic Pain. J. Knee Surg. 32, 1020-1023 (2019).

7. Kerver, A. L. A., Leliveld, M. S., den Hartog, D., Verhofstad, M. H. J. \& Kleinrensink, G. J. The surgical anatomy of the infrapatellar branch of the saphenous nerve in relation to incisions for anteromedial knee surgery. J. Bone Joint Surg. Am. 95, 2119-2125 (2013).

8. Singh, J. A., Mahowald, M. L. \& Noorbaloochi, S. Intraarticular botulinum toxin A for refractory painful total knee arthroplasty: a randomized controlled trial. J. Rheumatol. 37, 2377-2386 (2010).

9. Kachar, S. M., Williams, K. M. \& Finn, H. A. Neuroma of the infrapatellar branch of the saphenous nerve a cause of reversible knee stiffness after total knee arthroplasty. J. Arthroplasty 23, 927-930 (2008). 
10. Dellon, A. L. Partial joint denervation Il: knee and ankle. Plast. Reconstr. Surg. 123, 208-217 (2009).

11. Riegler, G. et al. Cutaneous nerve fields of the anteromedial lower limb-Determination with selective ultrasound-guided nerve blockade. Clin. Anat. (2020). doi:10.1002/ca.23582

12. Riegler, G. et al. Ultrasound Anatomic Demonstration of the Infrapatellar Nerve Branches. Arthrosc. J. Arthrosc. Relat. Surg. Off. Publ. Arthrosc. Assoc. North Am. Int. Arthrosc. Assoc. 34, 2874-2883 (2018).

13. Gunaratne, R. et al. Patient Dissatisfaction Following Total Knee Arthroplasty: A Systematic Review of the Literature. J. Arthroplasty 32, 3854-3860 (2017).

14. Grosu, I., Lavand'homme, P. \& Thienpont, E. Pain after knee arthroplasty: an unresolved issue. Knee Surg. Sports Traumatol. Arthrosc. 22, 1744-1758 (2014).

15. Rice, D. A. et al. Persistent postoperative pain after total knee arthroplasty: a prospective cohort study of potential risk factors. Br. J. Anaesth. 121, 804-812 (2018).

16. Koch, G. et al. Anatomical risk evaluation of iatrogenic injury to the infrapatellar branch of the saphenous nerve during medial meniscus arthroscopic surgery. Surg. Radiol. Anat. 39, 611-618 (2017).

17. Walshaw, T., Karuppiah, S. V \& Stewart, I. The course and distribution of the infra patellar nerve in relation to ACL reconstruction. Knee 22, 384-388 (2015).

18. Pivec, C. et al. Novel Demonstration of the Anterior Femoral Cutaneous Nerves using Ultrasound. Ultraschall Med. (2018). doi:10.1055/s-0043-121628

19. Mochizuki, T., Akita, K., Muneta, T. \& Sato, T. Anatomical bases for minimizing sensory disturbance after arthroscopically-assisted anterior cruciate ligament reconstruction using medial hamstring tendons. Surg. Radiol. Anat. 25, 192-199 (2003).

20. Orhurhu, V., Urits, I., Grandhi, R. \& Abd-Elsayed, A. Systematic Review of Radiofrequency Ablation for Management of Knee Pain. Curr. Pain Headache Rep. 23, 55 (2019).

21. Ahmed, A., Arora, D. \& Kochhar, A. K. Ultrasound-guided alcohol neurolysis of lateral femoral cutaneous nerve for intractable meralgia paresthetica: a case series. Br. J. pain 10, 232-237 (2016).

22. Dass, R. M. et al. Alcohol neurolysis of genicular nerve for chronic knee pain. Korean J. Pain 32, 223227 (2019).

23. Ellis, J., Schneider, J. R., Cloney, M. \& Winfree, C. J. Lateral Femoral Cutaneous Nerve Decompression Guided by Preoperative Ultrasound Mapping. Cureus 10, e3652 (2018).

24. Osorio, J. A. et al. Ultrasound-guided percutaneous injection of methylene blue to identify nerve pathology and guide surgery. Neurosurg. Focus 39, E2 (2015).

25. Gofeld, M., Bristow, S. J., Chiu, S. \& Kliot, M. Preoperative ultrasound-guided mapping of peripheral nerves. J. Neurosurg. 119, 709-713 (2013).

26. Lewis, G. N., Rice, D. A., McNair, P. J. \& Kluger, M. Predictors of persistent pain after total knee arthroplasty: a systematic review and meta-analysis. Br. J. Anaesth. 114, 551-561 (2015). 
Figures

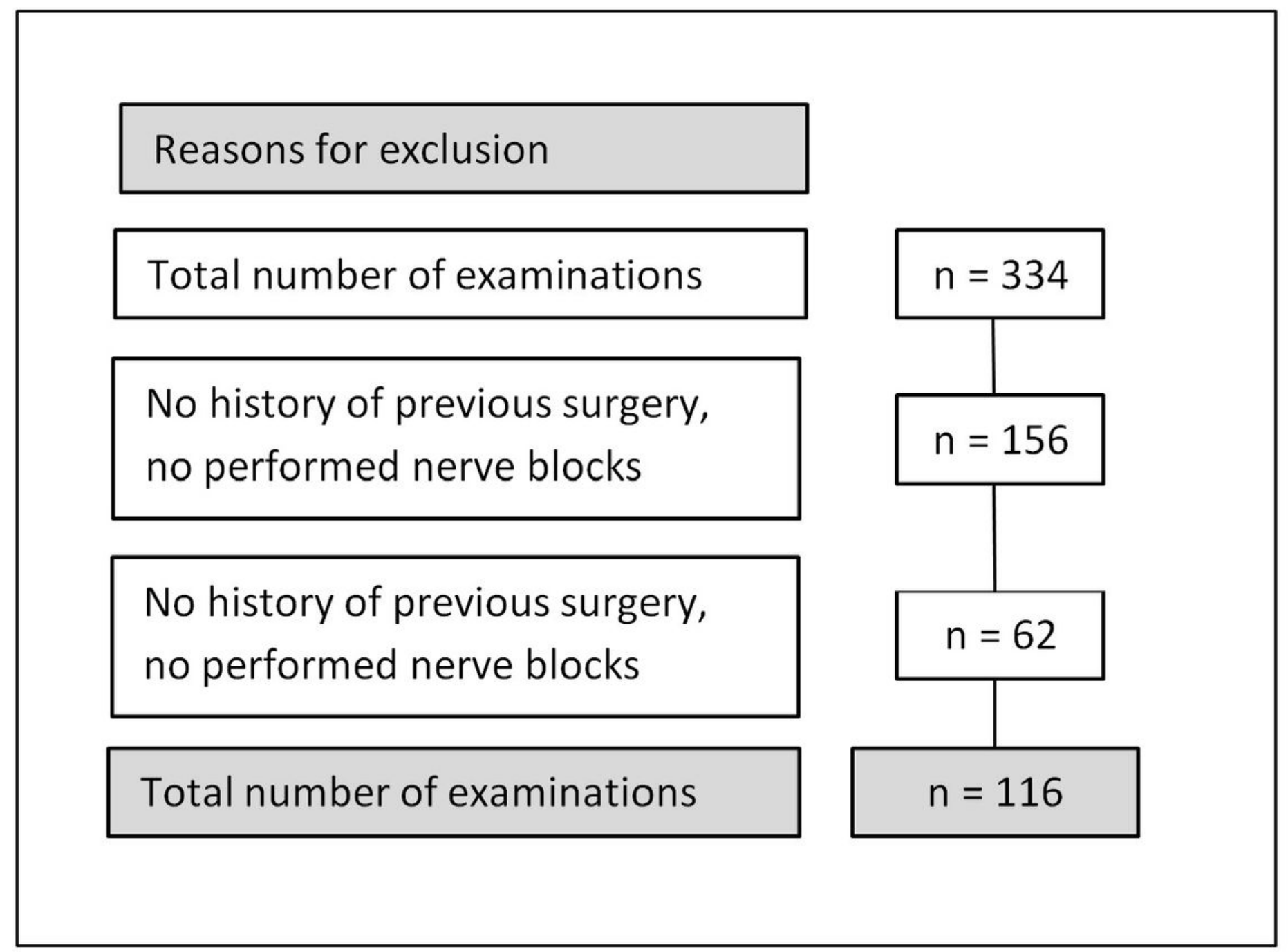

Figure 1

Study flow chart and exclusion criteria 

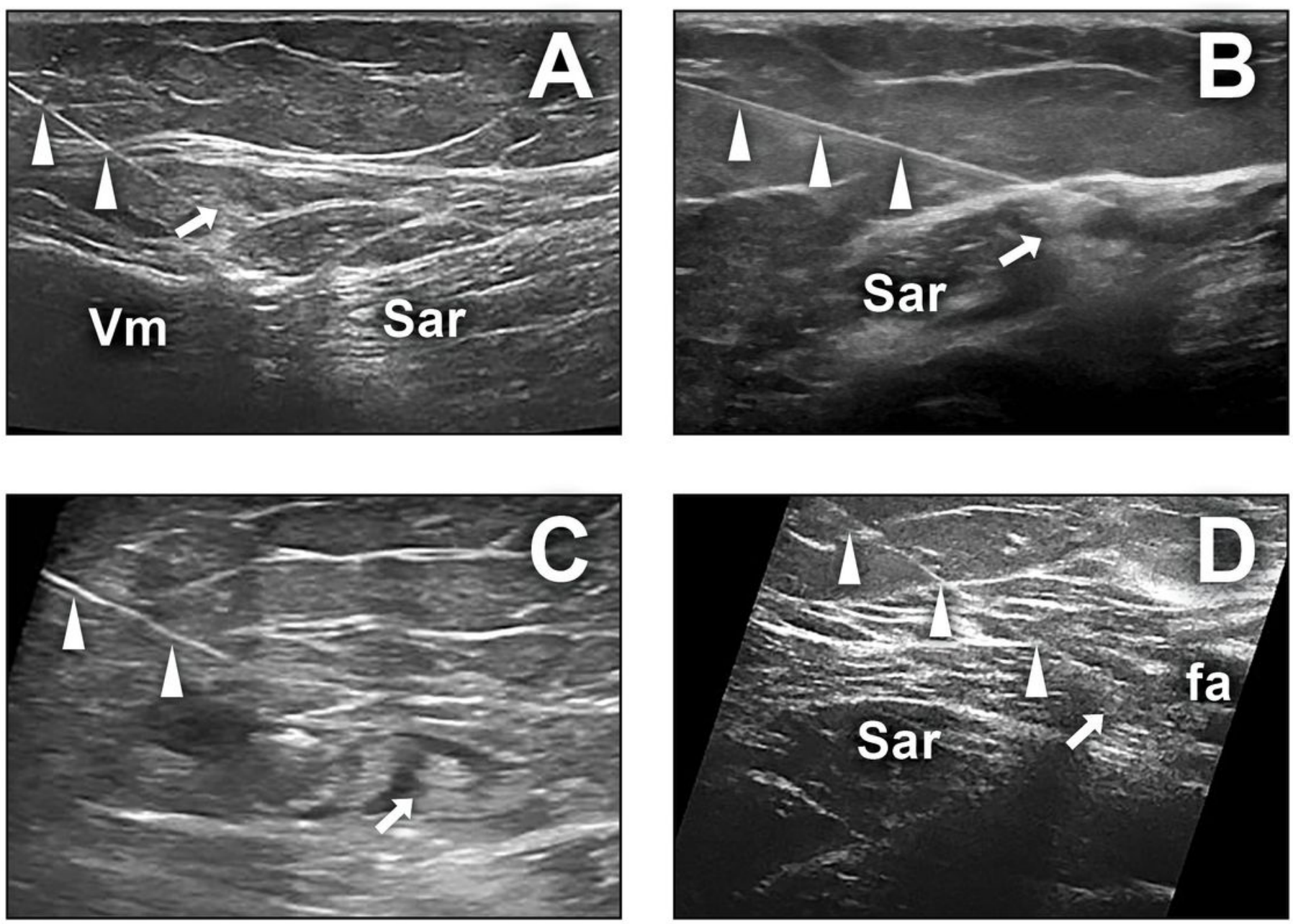

Figure 2

Examples of ultrasound-guided nerve blocks (USGB) in four different patients. A, USGB of the medial branch of the anterior femoral cutaneous nerve in a patient's right leg approximately $15 \mathrm{~cm}$ cranial of the knee joint; B, USGB of the infrapatellar branch of the saphenous nerve at the posterior edge of the sartorius muscle in a patient's right leg approximately $15 \mathrm{~cm}$ cranial of the knee joint; C, USGB of the infrapatellar branch of the saphenous nerve in a patient's right leg at the level of the medial knee joint; $D$, USGB of the saphenous nerve of a patient's right leg just $5 \mathrm{~cm}$ distal of the origin of the femoral nerve. Abbreviations: White arrowheads: needle, Vm: vastus medialis muscle, Sar: sartorius muscle, fa: femoral artery, White arrow: nerve. 

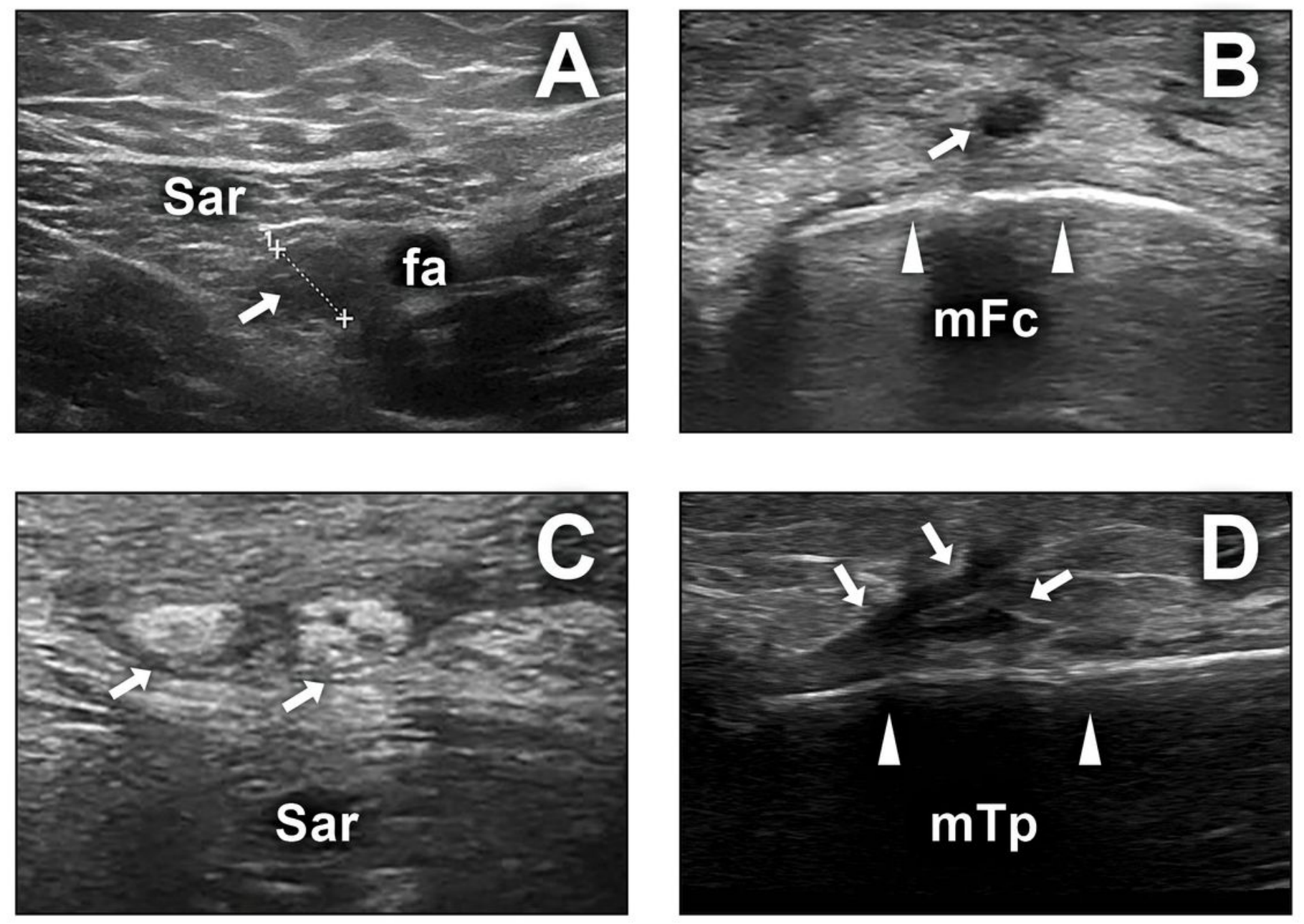

\section{Figure 3}

Examples of morphological nerve changes. A, Neuroma of the saphenous nerve in a patient's right proximal lower leg; B, neuroma of a infrapatellar nerve branch in a patient's left knee; C: nerve swelling (hyperechoic external epineurium) surrounded by hyperechoic scarring in a patient's left infrapatellar nerve branches; D, scar (hypoechoic) surrounding a right infrapatellar nerve branch. Abbreviations: White arrowheads: needle, Sar: sartorius muscle, fa: femoral artery, White arrow: nerve; mFc: medial femoral condyle; mTp, medial tibial plateau. 